\title{
Formation Stories and Causality in Sociology*
}

\author{
Daniel Hirschman \\ University of Michigan \\ dandanar@umich.edu \\ Isaac Ariail Reed \\ University of Colorado \\ isaac.reed@,colorado.edu
}

\begin{abstract}
:
Sociologists have long been interested in understanding the emergence of new social kinds. We argue that sociologists' formation stories have been mischaracterized as non-causal, descriptive, or interpretive. Traditional "forcing cause" accounts describe regularized relations between fixed entities with specific properties. The three dominant approaches to causality-variable causality, treatments and manipulations, and mechanisms - all refer to forcing causes. But formation stories do not fit the forcing causes framework because accounts of formation violate the assumptions that ground forcing cause accounts and instead emphasize eventfulness, assemblage, and self-representation. Yet these accounts are, we argue, fundamentally causal. In particular, formation stories provide the historical, empirical boundaries for the functioning of forcing cause accounts. We catalog the breadth of formation stories in sociology, and use examples from diverse literatures to highlight how thinking of formation stories as causal accounts can improve our understanding of the relationship of history and culture to causal analysis.
\end{abstract}

* Authors listed in alphabetical order; both authors contributed equally. We would like to thank Natalie Aviles, Beth Berman, Julian Go, Nathan Lauster, Carly Knight, Greta Krippner, Stephen Morgan, Alvaro Santana-Acuña, Taylor Spears, Lyn Spillman, and Nick Wilson for helpful comments on earlier drafts. Previous versions of this paper were presented at the Eastern Sociological Society (Boston), and at the Sociology in Progress Speaker Series at Colorado State University. Direct communications to Daniel Hirschman, c/o Department of Sociology, 500 S. State St., Ann Arbor MI, 48109. Preprint version. Final version published in Sociological Theory and available here: http://stx.sagepub.com/content/32/4/259.abstract 


\section{Introduction: The Historical and Variable Ontology of the Social}

It is a basic principle of sociological theory that social things (from organizations to identities to racial groups to states) are real because they are constructed. In other words, the reality of social things is a function of the work done to produce and sustain them. An important corollary to this principle is the historicity of social kinds. The ontology of the social is historically variable; the kinds of social things that exist now did not always exist and may not always exist in the future. ${ }^{1}$

Stated as such, this argument may seem clear enough, if controversial. Yet when we examine examples - the invention and diffusion of individual agency, the emergence of modern states, the construction of scientific facts and the techniques associated with them-we quickly see that, although sociologists in various subfields have long studied the coming into existence of particular social kinds, entities, or objects, social theorists have paid relatively little attention to the

\footnotetext{
${ }^{1}$ Much of what is sometimes called metatheory and sometimes called ontology creates an account of the basic entities that exist in the social world, with the implicit or explicit claim that these entities are transhistorical (e.g. calculating human actors, microinteractions, etc.). This is often opposed by an approach to theory and metatheory that insists on its "downward shift" into history, culture, etc. (Alexander and Seidman 2008). Our goal here is to turn this opposition between theory as basic ontology and theory as history into a more rigorous understanding of how historical changes in social kinds bound abstract theoretical work in sociology, and thus should themselves be subject to empirical study and theoretical analysis.
} 
structure and logic of such studies. ${ }^{2}$ We propose the label "formation stories" to characterize these studies, as they follow the formation of new social kinds as a historical process. Drawing on insights from historical sociology and science studies, we argue that formation stories offer explanatory, causal claims.

Although formation stories should be understood as causal, they fit poorly into the dominant modes of causal argument in sociology, which can be collectively characterized by the metaphor of "forcing." Forcing cause claims trace the movements of pre-defined objects into pre-defined outcomes, and argue that certain objects or interactions cause other objects to realize a certain outcome. In contrast, formation stories explain how social things come to be stable enough to force or be forced. ${ }^{3}$ Thus, formation stories establish historical boundaries past which forcing cause claims likely will not travel.

By characterizing formation stories as causal, but not as mechanisms, treatments, or robust associations, we can begin to produce new criteria and methods for making formation claims more rigorous. Our aim here is thus similar to Mahoney (2000), who attempts to clarify the logic of path dependence

\footnotetext{
${ }^{2}$ This inattention has been accompanied by a lot of writing at cross-purposes and conceptual controversy, for example the extended definitional debate about the "agency" or "causal power" of nonhumans in Actor Network Theory (see Sayes 2014 for a review).

${ }^{3}$ Recent work in scientific realism has emphasized the way entities have "causal powers" (Groff and Greco 2013). In this language, the formation of stable objects could be referred to as the invention or reshaping of an object or kind's causal powers.
} 
arguments. We argue that formation stories are an important class of sociological argument with their own peculiar characteristics and which thus necessitate further theoretical and methodological reflection. By treating formation stories as causal accounts, we argue that such stories have been simultaneously common in sociology and commonly misunderstood. On the one hand, they have been written off (or occasionally, in bouts of epistemic counterculturalism, valorized) ${ }^{4}$ as merely descriptive, rather than explanatory claims. On the other, this dismissal has left them underscrutinized, because formation stories have not been subjected to the rigorous evaluations, careful skepticism, and extended debates that other causal claims have faced.

Although formation is a messy historical process, we can discern some important lines of commonality across formation stories. In particular, we highlight the importance of both sociotechnical assemblages and actors' own understandings of the social world in the process of formation. This synthesis helps to clarify the distinction between formation stories, which trace historical shifts in the sets of objects actually existing in the social world, and the more typical social scientific analysis of concepts (cf. Sartori 1970, Goertz 2006). Social science has long had a tradition of examining "concept formation" and the construction of the object of analysis by the investigator, with particular interest

\footnotetext{
${ }^{4}$ The clearest and loudest of these voices is Andrew Abbott, who argues that "we who like to imagine ourselves responsible for the public's knowledge of society despise description" (Abbott 2001: 121). See also Abbott 2007.
} 
in both the intellectual origins of and scope conditions for the application of welldefined concepts (cf. Somers 1995). ${ }^{5}$ Furthermore, it is also well known that the work of social scientists may itself help to constitute or reconstitute social kinds. However, our overall argument here is that in formation stories, the mode of inquiry is historical rather than definitional. Thus the conceptual work we propose to do in this paper would point less towards debates about the definition of democracy as an analytical object in contemporary political sociology, and more towards debates about the emergence of democracy as an actually existing social kind. Put another way, this article is itself is a work of conceptual analysis of the concept of "formation stories." The exemplars we analyze, however, are empirical works that trace historically the formation of new social kinds, not works of conceptual definition and operationalization.

Clarifying our understanding of formation stories as causal accounts offers three interrelated benefits. First, we hope our analysis will lead to better formation stories, and particularly, better criticism and evaluation of the causal logic of such arguments. Second, by foregrounding the role of formation stories, we shed light on the limitations of traditional forcing cause arguments. Taking formation stories

\footnotetext{
${ }^{5}$ Though the idea that some sort of analytical/theoretical work is done to construct an object of empirical analysis can be found across the social sciences, sociology in particular traces this dynamic - and the impact of historical work on sociological theory_to Max Weber's (1949) 'Objectivity in Social Science and Social Policy”. See Outhwaite (2010), Oakes (1990), Ringer (1997), and Bourdieu, Chamboredon, and Passeron (1991).
} 
seriously entails not only the typical philosophical modesty about causality, but also the setting of empirical, historical boundaries to our forcing cause claims. Third, understanding formation as a causal process helps to disentangle arguments about the causal influence of culture. Culture (in its various manifestations) may act as a forcing cause, but it may also act through the formation or transformation of social kinds.

The argument proceeds as follows. We begin by reviewing the three dominant approaches to causality in sociology and arguing that they are usefully characterized as forcing causes. We then synthesize existing theoretical treatments of events, boundaries, assemblages, and looping kinds to make sense of the causal logic of formation stories. To begin the project of identifying regularities in formation stories, we then provide a typology of formation stories. We conclude with a return to the analytical payoffs of separating formation stories from forcing cause accounts for our understandings of the role of history and culture in sociological analysis.

\section{Forcing causes in sociology}

Here, we summarize the three most active, well-established approaches to causality in sociology and argue that all three fall under the rubric of "forcing causes." These are, briefly, "variable causality," "manipulation and treatment," and "mechanisms" In identifying these approaches, we rely on a rich literature on causal modeling, and in particular on similar distinctions drawn by Goldthorpe 
(2001). While these literatures disagree on many points, and the second and third arguably emerged in reaction to the perceived flaws of the first, we emphasize certain important commonalities which limit the scope of forcing cause claims in a way that points to the need to theorize formation stories as causal.

\section{Variable Causality}

In the classic account of causality developed in the post-war era of sociology, causal claims functioned by eliminating suspected sources of spurious correlation, thus establishing a "robust dependence" of an effect, $\mathrm{Y}$, on a cause, $\mathrm{X}$ (Goldthorpe 2001). An exemplar is Blau and Duncan (1967): Father's education causes son's current occupational prestige both directly and indirectly, through son's education and first job. This approach to causation is familiar from the essential statistical-sociological operation of "controlling for" variables in standard linear regressions and related techniques. Variable causality has been mobilized in many different social scientific disciplines, and, philosophically speaking, participates in the long tradition of linking causation to prediction (Hempel 1965).

Variable causality has weathered a great deal of criticism. Here, we highlight some of the key assumptions that underwrite the approach. Abbott (2001) catalogued six stated and implicit, but potentially problematic, assumptions of the "general linear reality" approach that characterizes most studies in this tradition, which he labeled "sociological causalism." We focus here 
on the first two assumptions: (1) that the social world consists of fixed entities with varying attributes and (2) that causality flows from large to small, from general trends to specific outcomes. Abbott's (1988: 172-173) original formulation emphasized the frailty of this first assumption in contexts where entities "change through birth, death, amalgamation, and division." Later work went further to charge that sociological causalism assumed fixed and unchanging meanings and in so doing ignored the potential for meanings, and thus the social kinds that they help to form, to change:

Most important, the 'meaning of parameters' can itself change for strategic reasons, that is through deliberate action of actors in a system. ... we all believe that one of the central, basic human actions is to redefine something, so that the very shape of the present, perhaps even the identities of the actors in the present, can be made new. (Abbott 1995)

The second assumption identified by Abbott (1988: 173) is that of "monotonic causal flow," the idea that causality flows from "big to small (from the contextual to the specific) or between attributes of equivalent size." ${ }^{\prime 6}$ Researchers are asked to investigate how general social trends shape particular outcomes that are assumed to be smaller than the trends that combine to produce them. Causality never flows from "the arbitrary to the general, from the minor event to the major development." (Abbott 1988: 173) Here, Abbott prefigures Sewell (2005) in

\footnotetext{
${ }^{6}$ It is important to note that Abbott's understanding of "context" here is related to his understanding of "grand immanent forces," rather than what many sociologists and anthropologists would identify as the "local context" for the working out of a more generalizable cause or mechanism.
} 
criticizing sociological causalism for its inattention to eventfulness, and thus for the possibility that seemingly "small" perturbations can have seemingly "large" consequences. A related criticism of monotonic causal flow comes from the science studies tradition. In contrast to the assumption that there are levels in the social world, and that causality flows in one direction between them, actornetwork theory suggests that researchers start with a flat ontology wherein no actor is inherently "bigger" or "at a higher level" than any other, and then trace the process by which some actors become big (Callon and Latour 1981). Both Abbott and Callon and Latour's critiques share the suspicion that standard sociological accounts misperceive social causality as constituted by "grand immanent forces" (Abbott 2001: 112).

While later work - including Abbott's own - partially addressed these concerns with variable causality, Abbott's first objection remains, in our view, the most important and the least addressed. Variable causality assumes fixed entities with varying attributes. An attribute, in this view, has an effect on another attribute: one's education affects one's income; one's race affects one's wages. Two more recent approaches to causality abandon this focus on variables as causes while maintaining the assumption of fixed entities. The first such tradition shifts our attention from variables to treatments.

\section{Manipulation and treatment}


In a series of influential works (Holland 1986, Heckman 2005, Morgan and Winship 2007), statisticians and social scientists have advocated for a reconceptualization of causality in a more strictly experimental vein. To understand causality, they argue, one must understand the effect of an intervention on a set of subjects, with that "intervention" - however it is generated - understood as the cause. In this approach, the first best option to establish the effect of a cause is an actual randomized controlled trial. The second best is some sort of quasi-experimental technique like regression discontinuity, propensity score matching, or instrumental variables. All of these recognize the "fundamental problem of causal inference" (Holland 1986: 947) an entity cannot be in both the control group and the treatment group. Much of the intellectual innovation in this tradition is built up around attempts to deal with this problem well enough to make causal claims.

Notably, a strict reading of this approach says that causality can only be discussed, in principle, for something that can be experimentally manipulated. This manipulation-based understanding of causality fits cleanly with program evaluation-type research where individuals can be assigned into a treatment or control group (receiving a certain intervention or benefit). ${ }^{7}$ However, in so doing

\footnotetext{
${ }^{7}$ Morgan and Winship (2014: 434-436), drawing on Woodward (2003), argue that the manipulability requirement is not as binding as critics argue, and in fact pushes researchers to clarify complex causal arguments. For example, the causal effect of race can be broken apart into different elements, such as the effect of
} 
it tends to narrow the set of questions that can be legitimately answered in terms of a causal effect in the quest to avoid the dreaded problems of endogeneity and selection bias. More importantly for our purposes, note again a basic assumption - that the entities being treated are fixed, as are the treatments themselves. Here again we encounter the pushing and pulling of extant social realities, in this case with the pushing and the pulling done by the experimenter or intervening agency.

In the treatment-based approach, causality here flows from the manipulation itself: if we push people into group A instead of group B, what happens? This framing leads naturally to criticism that, in its imitation of practical-scientific interventions, this understanding of social causality grants a great deal of intentionality and agency to the experimenting scientist, but none to the entities that are experimented upon (Goldthorpe 2001: 7-8). This problemand the attendant need to render social causality commensurate with at a concept of the social actor as a knowledgeable agent—has been one important inspiration for the third approach to forcing causality.

\section{Mechanisms}

employers perceiving potential employees to be black instead of white, which is separate from the causal influence on educational attainment of wealth disparities between black and white families, and so on (see also Sen and Wasow 2014). Even granting this broader concept of manipulability, however, research in this tradition still requires a fixed menu of treatments and outcomes. 
The last major approach to forcing causality, usually referred to as a mechanistic or social mechanisms approach, is often presented as a radical departure from variable and experimental causality. We identify two broad variants of this mode of understanding causality, focusing on the ways in which mechanism is "an irreducibly causal notion" that "refers to the entities of a causal process that produces the effect of interest" (Hedstrom and Ylikoski 2010: 50). ${ }^{8}$

In the mechanistic view, causality is a generative process. Instead of conceptualizing variable $\mathrm{X}$ or treatment $\mathrm{T}$ as a cause of $\mathrm{Y}$, this approach posits a causal process, $\mathrm{M}$, which moves a piece of the social world from $\mathrm{X}$ to $\mathrm{Y}$, and argues that social research should proceed by identifying and characterizing these causal processes. ${ }^{9}$ Something happens to "trigger" a mechanism or make it "work" (the input) and the process then produces the effect of interest (the output or outcome).

\section{Mechanisms as Microfoundations}

\footnotetext{
${ }^{8}$ Epistemologically, interest in "generative process" and "social mechanisms" is a part of several high-profile approaches to social science, such as rational-choice theory, analytical sociology, various small-n approaches in comparative-historical sociology and comparative politics, and critical realism. Not surprisingly, then, the definition of and use of the concept of mechanism is extremely contested in the literature.

${ }^{9}$ Thus, as James Mahoney explains, "[i]n this literature, causal mechanisms are often understood as intervening steps between an initial cause and a final outcome" (2012: 10). Given this, one way to understand mechanisms, then, is just as intervening variables; this can also connect to the treatment understanding of causality and its use of Directed Analytic Graphs (Knight and Winship 2013). Mahoney (2001) discusses these issues at length while providing an account of the broad origins of mechanistic thinking in sociology.
} 
Certain important strands of the mechanisms literature have attempted to use the concept of mechanism to (1) secure a clear set of "microfoundations" for causality in the social world, usually focused on individuals, their motivations, and the consequences of their interactions, and (2) link these foundations to the variable-based analyses that are recast as correlational descriptions which are then explained by mechanisms. That is, variable causality is reconceptualized as precise description (Savage 2009: 159-160), which is paired with a mechanisms approach to causality so as to produce a rigorous social science based on quantitative description and mechanistic explanation. (Goldthrope 2007: 123-124).

Some of this work is directly allied to the view of rational action as the necessary microfoundations of sociology (e.g. Coleman 1994), while other strands call for a broader understanding of actors and action. A well-known variant of this latter type is Peter Hedstrom's (2005) model of Desires, Beliefs and Opportunities. Despite opening up a broader conception of the actor than rational choice varieties, these approaches still rely on the fixed entities assumption. Hedstrom explains:

Mechanisms consist of entities (with their properties) and the activities that these entities engage in, either by themselves or in concert with other entities. These activities bring about change, and the type of change brought about depends upon the properties of the entities and how the entities are organized spatially and temporally. (Hedstrom 2005: 25).

This microfoundational approach to causality clearly articulates an understanding of the social world as a series of regularized processes in which well-defined, real 
entities with specific properties push and pull on each other and aggregate to produce "outcomes." One goal of this approach to causal mechanisms, then, is parsimony - to discover a relatively small set of underlying mechanisms with which one can explain a wide variety of phenomena that "on the surface" appear to be different.

\section{Mechanisms as Components of Conjunctures}

Perhaps reacting to these aspirations for parsimony, historical sociologists have criticized the microfoundations approach to mechanisms and advanced their own alternative. This alternative is developed via a theoretical language that departs explicitly from the variable approach to causality, and which views the merging of variable and mechanistic causality as mistaken. Instead, this approach focuses causal analysis on the conjuncture of different mechanistic processes. Steinmetz $(1998: 176,180)$ characterizes society or social life as an "open system" (cf. Bhaskar 2008: 107-131), and thus reconceptualizes historical contingency as the idea that "complex events are codetermined by constellations of causal mechanisms" (Steinmetz 1998: 177; for a nice visual representation, see 178). This formulation is particularly useful for producing an account of massive, rare, historical events (such as the French Revolution) which can be parsed into a conjuncture of mechanistic processes, with the understanding that, even if the conjuncture of such processes is relatively rare, the processes themselves can be found throughout history (Tilly 1995b). In this way, revolutions become more like 
floods than ocean tides (to use Tilly's evocative metaphor): they are rare events, but ultimately analytically separable into the mechanisms that combine to bring them about (Tilly 1995a: 1601).

Key to this way of thinking is the idea that a mechanism can even be triggered without bringing about its normal effects. In a complex causal conjuncture, other mechanisms can intervene, override, or combine with that mechanism to produce "unexpected" outcomes. Thus, instead of associating causality with the establishment of a robust association between observables, this work locates cause in the "real causal powers" of social mechanisms (Groff and Greco 2013).

So, mechanisms are defined as emergent causal powers of related entities within a system, which indicates (1) their existence at several different "levels" of reality (i.e. not just "micro"), (2) the complex way they exist as relations between various entities, and (3) that their causal powers are context dependent vis-à-vis the broader social system of which they are apart. Thus, for example, "a patent of nobility may have very considerable causal powers in one socio-historical context (a feudal society) but relatively little in another (a capitalist society), and a very opposite one in yet another (a communist society)" (Gorski 2009: 162). Mechanisms are thus, in this view, "space-time specific," "mutable," dependent upon human agency, and also "concept dependent," though they are not reducible to the "cultural concepts" that help make them up (Gorski 2009: 150, 164-165; for 
an example see Steinmetz's [2007a: 26-27] critique of Edward Said's cultural interpretation of colonialism).

There is thus, in this approach, relative consensus that accurate explanation with mechanisms requires investigators to pay attention to the context in which a given mechanism works. This stance accords with a more general discontent about the highly acontextual approach to causality that, in the post-war era, became "hegemonic in American sociology" (Abbott 2001: 113). Thus the idea that a social mechanism — or any other forcing causal relationship — has to be adequately contextualized so as to produce accurate, predictive, or "rich" explanation has been extensively developed. Given this, however, less attention has been paid to whether the notion of mechanisms adequately expands the sociological repertoire, and exhausts the space of causal reasoning necessary for sociological inquiry.

\section{Mechanisms as Forcing Causes}

While diverse and contested, the developing mechanisms literature still conforms to a basic understanding of "causes as forces" (Lakoff and Johnson 1999). The "causal powers" attributed to mechanisms, or conjunctures of mechanisms, derive from entities, their properties and the interactions and relations between these entities (Groff and Greco 2013). Furthermore, whether a given theory of mechanisms attempts to "reduce" causality to a fundamental level, or, alternately, embraces "explanatory a-reducibility" (Gorski 2009: 149), both 
approaches assume a stratified, layered ontology of human social life which occurs in "levels" (usually running from "micro" to "macro"). Hence Stinchcombe's more epistemological definition of mechanisms holds across many, ontologically divergent, accounts of mechanistic causality: "A mechanism is a piece of scientific reasoning... which gives knowledge about a component process" of another, ordinarily higher-level theory (1991: 367).

So, the extended debates in the mechanisms literature-including debates about variable ontologies, meso-to-meso explanation, and differences between social and natural mechanisms introduced by the way social mechanisms are "concept-dependent" - are underwritten by a key commonality: all of this literature thinks of mechanisms as configurations of entities with causal powers, that can be identified as present or absent, and thus are in some sense "modular" (for a strict definition, see Woodward 2003: 48). ${ }^{10}$

However, at the cutting edge of thinking about mechanisms, and particularly in the strand of the mechanisms literature that has reacted against the "microfoundations" approach to mechanisms, we can see a shift towards what

\footnotetext{
${ }^{10}$ This essential assumption may explain the foci of certain intense debates in the literature. For example, it accounts for why those who write in the critical realist idiom assign mechanisms to the sphere of the "real" (which produces the "actual" and the "empirical"), and thus engage in extensive debates on the "emergence" of real entities as higher levels of complexity. And it may account for why so much ink is spilled over the different "levels of analysis" in sociology, for the stakes of the "level of analysis" debate are very high if it turns out that "causal powers" can only be assigned to a subset of the total number of "levels."
} 
taking seriously the causal importance of formation. Both Philip Gorski (2009: 150) and George Steinmetz (2004, 2007b), for example, have argued for developing historically variable ontologies of the social as part of what they still sometimes identify as a mechanisms-based approach. This question of historically variable ontologies connects directly to our primary concern: how do all the objects and entities that cause things to happen in the social world come to be what they are, with certain meanings, "causal powers" etc.? That is, how do the forces in the social world come to possess a certain form or shape that makes them what they are? It is this issue that we hope to address with our concept of "formation."

Forcing cause claims offer an incomplete map of social reality

Though debate about causal inference and causal explanation has focused primarily on the differences between these three approaches, the similarities should by now be clear. First, these versions of causality all maintain the "fixed entities assumption" identified by Abbott. Even the "generative processes" approach associated with social mechanisms is not about the history of social kinds themselves, or about how entities subtly change their nature, meaning, or essential properties through some historical path. Rather, research that identifies mechanisms, and uses mechanisms to explain outcomes, is interested is how social objects that are real within the scope of the given analysis come together to force certain outcomes rather than others. 
Second, work on forcing causes is inclined towards - and indeed in some versions epistemologically requires - regularity as part of its theoretical coherence and explanatory power (see, e.g. Goldthorpe 2001: 10-11; Hedstrom and Bearman 2009: 5). This regularity requirement perhaps accounts for the appeal of mechanism as a metaphor for social process, since machines are renowned for, and useful because of, their regularity. More substantively, this emphasis on regularity recalls the rigor of scientific experiments - and in particular their repeatability — as the method of discovering the workings of the natural world.

Given the existence of social kinds of various shapes and sizes, and the tendency for such entities to act on the world in a regularized way, we agree that the pursuit of forcing causes is an intellectually rich and worthwhile endeavor. Furthermore, for a variety of normative reasons, investigating those entities and mechanisms that can be intervened upon or manipulated so as to produce a different (known) outcome or result can be especially rewarding. And, we have no doubt that forcing cause accounts can be, and often are, true explanations of certain social phenomena or outcomes. Yet, simultaneously, it is also the case that forcing cause accounts tend to underestimate the importance of their boundaries, which should be determined empirically, and to overestimate the stability of their results, leaving the shaping and reshaping of the entities in the social world to the 
side in their research. When it comes to forcing causes, that is, there is a danger of sliding "from the model of reality to the reality of the model" (Bourdieu 1990: 39). As a result, those aspects of social life that do not fit into these causal regimes are, if not ignored, reclassified under a variety of different categories that imply non-causal or non-explanatory research — as historical description, interpretive sociology, second-order interpretations, etc. This reclassification of claims about the formation of forces, kinds, and objects as non-causal is confusing and prevents rigorous methodological discussion about actual research practices. A good example of how this happens can be found in comparative-historical sociology. That subfield has often been anxious to define as causal those things that fit one of the definitions of causality discussed above, and thus characterize other aspects of the work of historical sociology as consisting of interpretation and description (for example: Mahoney and Rueschemeyer 2003: 22-23; Kiser and Hechter 1991; Quadagno and Knapp 1992; Calhoun 1998: 860-867; Ragin 1989: 31). And yet, a recent review argues that "to explain the origins of social order requires tracing the emergences of structures, institutions, or other collective properties that transform the causal relationships that dominated prior to the episode of ordering" (Clemens 2007: 534), and then notes how Michael Mann's explanation of fascism in terms of "social caging" involves a kind of epistemological shift: "Note the contrast between Mann's definition of the 
problem of fascism as a process of group emergence and other correlational analyses that address the question of who voted for the Nazis..."

We propose that this example is quite indicative of a broader trend, whereby a quite narrow methodological definition of causality is consistently being brought into contact with research that cannot make its (implicitly causal) claims fit into the definitions of causality that are articulated in standard methodology texts. In addition to creating a disjuncture between what sociologists say they are doing and what they are actually doing, this mismatch severely limits the capacity for critique and improvement within the community of inquiry that is concerned with formation stories.

\section{Theorizing Formation Stories}

Given the wide variety of sociological research that does in fact attend to formation, a broader conceptualization of causality is called for. We thus theorize the formation of objects, kinds, and forces to account for the way that new social kinds can come into being, and extant social kinds can be reshaped such that their properties or "causal powers" are fundamentally altered. Formation stories instantiate, we propose, four essential departures in the way causality is usually understood in sociology.

The first departure, drawing from Abbott (2001), is to relax the assumption of "fixed entities." As noted above, most sociological research makes strong assumptions about the fixity of social kinds. For example, a great deal of 
work charts the movement of individuals (or organizations, or nations, etc.) between fixed states: health researchers might focus on what kinds of people experience certain illnesses, while stratification research asks whose children manage to move up in the social hierarchy. The possible outcomes (sick or healthy, rich or poor) are known in advance and assumed to be relatively unchanging in significance. Classic works of historical sociology make similar assumptions about inputs (or "conditions"), outcomes, and the forces that move a "case" from one to the other-e.g. from a single location of sovereignty to different types of "revolutionary situations" (Tilly 1995b: 10-14). ${ }^{11}$ Formation stories, in contrast, embrace the variable ontology of the social and reject the assumption of fixed entities, proposing instead to trace how entities, outcomes, categories, and kinds came into being or we reshaped.

The second departure, drawing from Sewell (2005) is to treat history as eventful. One aspect of the messiness of social life is that some actions, accidents, decisions, and details are causally much more important than others, in terms of how they shape the future of social life from a certain point in time and space. Some occurrences change the fabric of social reality such that the possibilities for causal regularities — of the forcing cause variety — are different before and after

\footnotetext{
${ }^{11}$ One exception, noted by Abbott (2001:60), is demographic or ecological models that attempt to explain births and deaths. This exception reinforces the general problem though: we can start thinking about how to model the birth or death of an existing kind (individuals, organizations, etc.) but it is much harder to model the emergence of new kinds or the disappearance of an entire kind.
} 
what we then mark as the "event." Exactly how the event unfolded can dramatically alter the kinds of entities that emerge from the event. ${ }^{12}$ Events may be very short and dramatic, as in the classic case of the storming of the Bastille. But the idea of an eventful temporality goes a bit beyond this emphasis on key moments, to include a broader focus on disjuncture and rupture in history (cf. Foucault 1972). Of course, social theory has long been interested in history and eventfulness. Our point is more narrow but also more precise: how did certain occurrences, possibly minute, alter the sorts of entities that came into being, and the forcing cause relationships that those entities engage in?

The third departure, drawing from actor-network theory, is an emphasis on assemblages. Social kinds emerge through processes of assemblage, which mix together a heterogeneous collection of individuals, organizations, technologies, ideas, significations and more (Latour 2007). Formation stories apply this way of thinking to the emergence of new things-in-the-social-world, like "public opinion," and to the reshaping of extant social categories, like "whiteness" (Baker 1990, Osborne and Rose 1999, Omi and Winant 1994). So, for example, instead of thinking of "the economy" as the material substratum of social life, consider how "the economy" came into being as a technical object measured by the fluctuations

\footnotetext{
${ }^{12}$ Abbott also has a version of events, and he connects it to his critique of scientific causalism in American sociology, in so far as a focus on events, relationality and action is his proposed remedy for the "full-blown ANOVA concept of causality" (2001: 113); see especially Abbott 2001: 120-125; 209-239.
} 
in Gross Domestic Product (Mitchell 1998). Our point here is not just that "objects" are "constructed." Rather, our point is that existing social kinds are real entities with real consequences, but also with real histories, and that these histories are twisted and varied; to make a entity with the power to "force," we propose, requires a process of assemblage which does not fit neatly into the languages of $\{$ macro-, meso-, mirco- $\}$, \{culture, politics, economy $\}$, or $\{$ ideal, material $\}$. For this reason, tracing the formation of kinds, categories, and entities involves linking together actions, communications, various kinds of material stuff, and a wide variety of mediations, in a way that challenges traditional understandings of levels of analysis. This tracing can be termed the tracing of "assemblage" because it is relatively agnostic about the kinds of "stuff" that can come together in a formation story.

Fourth, and finally, we draw from Ian Hacking (2000, 2002), who emphasizes the unique characteristics of assemblages involving knowledge about people. Hacking names this approach "dynamic nominalism," and thus brings into the causal conversation the question of how representation has an impact on social life. Hacking emphasizes the ability of names (and, more broadly, knowledge and theories) to reshape the thing named — if and only if the thing in question is somehow aware of the name it is being given. This requirement of awareness differentiates arguments about the social construction of atoms from 
arguments about the social construction of autism (Hacking 2000). ${ }^{13}$ Autistic individuals, and their parents, friends, partners, teachers, and therapists all (potentially) know about autism (theories of autism) and change their behavior accordingly, choosing some treatments over others, joining some support groups over others, and so on (Eyal 2010). These individual changes in behavior have the potential to reshape the causal significance of the category - that is, what it means, for a diagnosed individual, family member, or psychiatrist to be autistic can change depending on how an important subset of people understand autism. On the other hand, atoms do not, in principle, care whether we think they look like plum pudding or mini-solar systems, and do not, in principle, change their behavior depending on our theories. Hacking's insights point to the potential for culture, in various forms, to play a critical role in formation stories.

${ }^{13}$ John Searle (1995: 1-58; 127-148) famously distinguished between the "intrinsic" and "observer-relative" properties or facts about objects, igniting much philosophical debate. One way to characterize our argument here is to say that most sociologists accept that many of their objects of study include observerrelative properties of objects, persons, etc., and that most sociologists also accept that what Searle characterizes as "collective intentionality" is a baseline from which sociologists work. However, it is perhaps too restrictive to insist debate continue at this level. As Neil Gross explains, Searle's basic formula, according to which social facts take the form "X counts as $Y$ in context $C$," tends to reduce the complexity of culture and history to the level of cognitive rules. Gross (2006: 54) writes "it is not clear that the metaphors, genres, and tropes that also play a significant role in our understanding of institutions, and hence in constituting those institutions as real, can be reduced down to rules of this sort... there is reason to doubt whether Searle's understanding of the nature of constitutive rules will seem adequate to many sociologists." 
To summarize, then, when we speak of formation stories, we refer to explanatory accounts of how social kinds are shaped, reshaped, or brought into being; in contrast to forcing causes, these stories take as their points of reference the non-fixedness of social entities, the eventfulness of social life, the emergence of social entities from processes of assemblage, and the dependence of such assemblage and non-fixedness on representation. These four departures provide guideposts for our analysis of existing research on formation: We will be interested in work that explains the emergence of new social kinds, breaks history into pieces based on events, traces messy, heterogeneous processes whereby new social kinds emerge, and which involves a concept of representation. As the reader will quickly see, the four departures are partially descriptive of existing research practices and partially prescriptive, intended to shape future work. The following section offers a simple classification of formation stories based on the kind of object being formed, and in so doing illustrates some of the theoretical payoffs of bringing together previously disconnected research traditions under the heading of formation.

\section{What gets formed?}

To this point our discussion of formation in sociology has been largely abstract, to match the existing methods discourse on causality. Here, however, we make our argument a bit more concrete by analyzing four types of formation stories, each of which is concerned with the formation of a different sort of object 
for sociological analysis: actors, meso-level social orders, sociotechnical objects, and macro-level cultural "atmospheres." ${ }^{14}$ To illustrate the first three types, we explore well-established areas of research, albeit ones that have not previously been recognized as subtypes of a larger class of arguments. The fourth type, cultural atmospheres, we consider as a possible extension of formation arguments, which could help address the complex problem of culture and causality in sociology. In what follows, we note how each type of formation story already draws on, or could benefit from explicit attention to, the theoretical departures identified above: the rejection of fixed entities, eventfulness, assemblage, and representation. The first two sections emphasize the breadth of formation stories already prominent within sociology. The third and fourth consider specific examples to highlight the relationship between formation stories and forcing causes.

\section{The formation of actors}

In many different areas of sociology, and certainly in sociological theory, the social world is understood to contain "actors" (individual or collective) that push and pull this way and that, and thus produce various outcomes (Alexander 1981: 114-122, Sewell 1992, Giddens 1979: 49-96, Hedstrom 2005: 34-66). Sociological theories built on the ability of actors to act as forcing causes are

\footnotetext{
${ }^{14}$ Of course, in any given account, there may be intertwining between the types: the formation of a meso-level social order may involve the formation of a new sociotechnical object or a new social actor, for example.
} 
constrained by our knowledge of the properties or tendencies of those actors. One response to this constraint has been to construct theoretical models rooted in clear assumptions about these properties and tendencies, as in the tradition of rational choice theory. An alternative line of research, however, treats actors' capabilities as an empirical and historical question, and aims to produce explanatory accounts of how actors came to be the actors that they are.

This alternative, empirical account of actors' properties is most familiar in the case of collective actors - groups of people that assert their "interests" as a whole, engage in contentious politics with states and other entities in their political environment, and attempt to force certain kinds of outcomes. Where do they come from? Why do they want what they want? How do collectivities with well-defined boundaries and clear demands form? This question is evident in classical sociology, as in Max Weber's skeptical response to socialist theorists wherein he suggested a series of barriers between an objective, shared "market situation" of individuals and communal action based on that collective interest (Weber 1958: 183, cf. Fourcade and Healy 2013). More recent work examines how the collectivities that engage in contentious politics come into being as unified entities - e.g. through constitutive boundary change (Tilly 2004) or the formation of collective identities that did not exist before (Polletta and Jasper 2001). 
Since Marx's own (admittedly teleological) account of the rise of the proletariat as a class for itself, the sociology of collective action has been in some way "eventful" in its recognition that the formation of a certain collective actor with certain collective properties can create a new set of social tensions and forceful relations in the world. However, the importance of assemblage has been much less clear in this literature.

Using the idea of assemblages to understand the emergence of collective actors as a case of formation may help clarify a tension surrounding the role of culture within studies of collective identity. In hindsight, we can see that in turning to collective identity, social movements scholars were really interested in two distinct sets of problems: first, how the "collectivities" that were engaged in politics came into being historically (something that had merely been taken for granted when the field of social movements studies turned from the analysis of mass politics to the analysis of the civil rights movement, see Polletta and Jasper 2001); and second, how "collective identity" allowed researchers to understand the non-instrumental basis of recruitment and strategy choice by already formed movements. The first problem leads to formation stories; the second considers how culture can act as a forcing cause via "framing" (Benford and Snow 2000). Thus we can see why the interest in formation does not really fit with the theoretical advances in the framing approach to social movements, for collective actors are assembled out of all kinds of "stuff" - stories, shared emotional 
experiences, economic resources, the built environment, and so on. The point of investigating such formation is, then, not to discern the specific causal power of framing, but rather to discern how a group came to be the group that it is (a group which, once formed, may indeed engage in "framing").

If the idea that collectivities have to be formed recalls the classic opposition between Marx and Weber, a more recent argument insists on the need for empirical formation stories about the individual actor itself. As Meyer and Jepperson explain, 'the modern 'actor' is a historical and ongoing cultural construction, and that the particulars of this construction should help to account for a number of specific features of actorhood, including anomalous and unnoticed ones." (Meyer and Jepperson 2000: 101) In their argument, the very existence of certain kinds of individuals, responsible in a certain way for their actions, is itself an empirical, historical story that has to be considered, precisely because it reveals essential aspects about individuals as agents that are important for building a theory of agents as what we would call forcing causes (for a similar argument about the historical production of individual actors, motivated very differently, see Foucault 1977).

For Meyer and Jepperson, the long arc of this story is a cultural tale about the devolution of agency from gods, spiritual powers, and an animated environment to individuals, organizations, and states, a kind of extended immanentization of cultural understandings of responsibility and activity: "Over 
time these exogenous forces (e.g. godly powers) have been relocated as authority immanent within society itself, enlarging social agency, relocating authority from god to church, from church to state, from church and state to individual souls and later citizens." (Meyer and Jepperson 2000: 101) Our point is not to affirm this specific formation story as the final or correct one, but rather to point out how this argument takes "agents" explicitly out of the realm of metatheory and conceptual definition, and instead makes them the subject of empirical explanation. This theme is perhaps the defining feature of formation stories: the existence of particular social things is not a matter for metatheoretical dispute, but rather empirical, and especially historical, explanation.

\section{Meso-level social orders}

Meyer and Jepperson locate their argument across the micro-macro spectrum, arguing that their formation story applies to individuals, organizations, and states as "actors." While there is much to be gained from collapsing the distinctions between different levels of analysis to highlight unifying features (including the mere fact that none is more natural, or less constructed, than another), it is not the only option. A particularly vibrant tradition of sociological research narrates the formation of meso-level social orders, which are not seen as actors, but rather serve as the environments in which actors (be they individuals, organizations, social movements, or states) act. 
Fields are perhaps the most iconic variety of meso-level social order. Studies of field formation examine empirically the coming-into-being of a space for action, with attention to the field's shape, scope, and relationship to other fields. For example, Barman (2013) documents how the field of "non-profits" emerged as foundations fought to be classified alongside the charitable organizations they funded, and thus to shield themselves from political scrutiny. This struggle took place through an influential classification system of tax-exempt organizations, which in turn produced a field of non-profit research and helped to legitimize this broader conceptualization that lumped the Ford Foundation and local soup kitchens into the same field. In a similarly framed argument, Tom Medvetz (2012) documents the "crystallization of the space of think tanks." Medvetz's explanation for the formation of the space of think tanks highlights a quite particular - but obviously, in an eventful sense, massively important "structural convergence" between technocratic organizations who needed to make their knowledge more open to the public, and activists who needed to become "experts" and thus have their own knowledge claims become more "closed." Given the influence of synthetic theoretical frameworks which emphasize the dynamics of fields such as Bourdieu (1984), DiMaggio and Powell (1983), and Fligstein and McAdam (2011), it is not surprising that research on the formation of fields has been an empirically successful enterprise. 
Beyond fields per se, Padgett and Powell's (2012) recent treatise on The Emergence of Organizations and Markets offers another elaborated research program on the formation of meso-level social orders. Inspired by a research tradition in economic sociology going back to White's (2008) influential work on "how social formations emerge," Padgett and Powell argue that new markets emerge out of the interactions of multiple networks, as logics and resources flow from one network into another and are repurposed. Through a series of case studies, Padgett, Powell and co-authors offer examples of novel organizational forms emerging out of existing, overlapping networks, such as the birth of the partnership system in renaissance Florence.

Thinking about meso-level social orders through the lens of formation stories makes sense of recent calls for some kind of alliance or peace between the sociologies of Pierre Bourdieu and Bruno Latour (cf. Eyal \& Buchholz 2010). Without delving all the way into constructing an opposition between these theorists and its possible solution, we can nonetheless see one way forward for such an alliance: research on field formation that emphasizes eventfulness and assemblage. A field emerges not only via criss-crossing strategies of individual and collective actors, but also through the appropriation of the built environment, the transfer of funds, the emergence of new communications technologies, and so on. Modern financial markets, for example, depend deeply on the speed of information transfer available, which in turn depends on the speed of light 
(MacKenzie et. al. 2012), and the field of think tanks required not only on the invention of a new symbolic space for the interpretation of experts and expert knowledge, but also the literal space of massive buildings positioned within less than a day's driving distance from Congress.

\section{Sociotechnical objects}

In terms of existing research, the emphasis on assemblage is best exemplified by formation stories about sociotechnical objects, which themselves often become part of formation stories about meso-level social orders and actors. The idea of object-formation of some sort of another is the focus of a diverse body of research in science and technology studies (e.g. Latour 1999, Daston 2000, Hacking 2002). This variety reflects in part the breadth of the category sociotechnical object itself, which spans everything from traditionally understood technologies like bicycles and bakelite (Bijker 1997), to diseases and diagnoses (Latour 1999, Hacking 2007), to technical versions of broad social phenomena such as public opinion manifested in polls and surveys (Igo 2007). What these stories have in common is an attempt to trace historically the emergence, or reshaping, of "thingness" in the sense of something that human actors designate denotatively, and attempt to manipulate, attack, leverage, etc. ${ }^{15}$ Thus sociotechnical objects is a category that refers to sociological research on the

\footnotetext{
${ }^{15}$ For a useful discussion of the concept of "object" in science studies, see Star 2010.
} 
formation of those social realities whose treatment by people is somewhat analogous to people's treatment of everyday material objects. ${ }^{16}$

In order to illustrate our broader arguments, here we examine here a single case of the reforming of a sociotechnical object in this sense: autism. We focus especially on the work of Gil Eyal (2013, see also Eyal 2010), and argue that his analysis usefully models the four departures critical for telling successful formation stories. In addition, Eyal (2013) illustrates the usefulness of formation stories for informing and bounding forcing cause accounts (both sociological and, in this case, biological).

Eyal (2013: 864) begins with a puzzle: how did autism grow from a relatively rare disease (affecting 1 in 2500 in 1989 in the United States) to an epidemic (affecting 1 in 88) in just two decades? Previous research on the question falls into one of two genres of explanatory account. First, naturalistic accounts search for biological causes of autism such as new environmental toxins, or the infamous and widely discredited vaccine-autism link (see Kirkland 2012). The second genre of account, broadly social constructionist in orientation, critiques the naturalistic accounts for ignoring the obvious changes in the ontology of autism that occurred in the late 20th century. These accounts emphasize changes in the diagnostic criteria beginning in the 1980s that opened

\footnotetext{
${ }^{16}$ These objects may be "actors" in the ANT sense (Sayes 2014), but not in the sociological sense; they may be constituted within fields of expertise or other meso-level social orders, but they are not co-extensive with those fields.
} 
up autism diagnoses to new kinds of individuals. Thus, the autism epidemic should be thought of as a process of diagnostic substitution and accretion: children who were previously diagnosed as mentally retarded or schizophrenic (or simply undiagnosed) are now instead labeled autistic.

While Eyal agrees with much of this second perspective, and especially the importance of diagnostic substitution, he argues that existing constructionist accounts fail to identify the real origins of the epidemic:

"Social constructionist explanations, by comparison, are more plausible but, ultimately, just as unsatisfying. They do not solve the puzzle of the autism 'epidemic' but merely push back the burden of explanation. If changed diagnostic criteria are the proximate cause, for example, the question obviously becomes why were they changed?" (Eyal 2013: 867)

In other words, social construction accounts are unsatisfactory without a good formation story to explain the shifts in diagnostic criteria. Did diagnostic substitution occur because we realized that autism was actually bigger than we previously thought (which restores the naturalist account) or because psychiatrists claimed increasing turf (medicalization) or something else entirely? We need an account, a causal explanation, of the shifts in the ontology of autism.

Eyal's account of the autism epidemic begins not with changes in the diagnostic criteria of autism, but rather a change in the institutional matrix through which we evaluate and treat mentally ill children. Specifically, Eyal argues that: 
[The] deinstitutionalization of mental retardation-a lengthy process that began in the early 1970s and lasted at least two decades - was a key cause leading to the autism epidemic... Deinstitutionalization erased the old categories that reflected the needs of custodial institutions 'feeble minded, mentally deficient, moron, idiot, imbecile' while creating instead a new institutional matrix - community treatment, special education, and early intervention programs - wherein autism could be identified, differentiated, and multiplied. (Eyal 2013: 867-868)

Deinstitutionalization is explicitly a key feature of the causal explanation of the autism epidemic. But what kind of a cause is it? For Eyal (2013: 868), deinstitutionalization was an event in a causal formation story (an "episode" in a sequence of reiterated problem solving), not a mechanism, variable, or treatment. From this episode of deinstitutionalization, Eyal traces a messy assemblage of actors and tools that make visible and transform autism from a tightly defined, and very limited in reach, network in the 1940s to a widespread and diffuse grouping of experts, parents, self-identified autistic individuals, checklists, treatment protocols, and funding streams. For example, by creating and distributing a simple checklist for autistic symptoms, psychologist Bernard Rimland managed to solicit information from thousands of parents, and in turn connected these parents to newly proposed behavioral therapies, growing the "autism matrix."

Although Eyal's central concern is a better understanding of the role of experts and expertise and not a challenge to sociology's understanding of causality, we can use his account of the autism epidemic to understand formation 
stories more generally. In addition to highlighting the role of events, culture, and messy assemblages in the formation and transformation of autism and the autism epidemic, the contrast between Eyal's formation story and forcing causal research on autism highlights how formation stories can usefully inform forcing cause accounts. Note that broadly social constructionist research may still fit inside traditional forcing cause approaches. For example, Liu et al (2010) offer a mechanisms account of the diffusion of autism diagnoses through social networks. Following Eyal's critique, however, we agree that such an account, while useful, on its own fails as an explanatory account of the autism epidemic because it cannot explain why autism suddenly became amenable to social diffusion in the 1980s. Eyal's formation story explains precisely that.

\section{Macrocultural "atmospheres"}

Could the formation story be extended beyond these three types? We do not regard our classification as exhaustive, and in that spirit we offer a more exploratory suggestion: that what are often understood as broad, macrocultural interpretations be reconsidered as a type of formation story explanation. To propose this, we use an iconic example in an intentionally provocative way.

It is widely agreed that a fiscal crisis precipitated the French Revolution. And yet, France had experienced fiscal crises many times before. What was different about the 1780s? Historians point to change in the cultural setting of the late 18th century, documenting the decreased legitimacy of the King vis-à-vis his 
famous grandfather (Darnton 1996). This new "cultural atmosphere" into which the fiscal crisis of the French state intruded can be rendered more precise via an examination of the formation of public opinion.

In "Public Opinion as Political Invention," Keith Baker (1990) traces the formation of public opinion as a politically relevant reality in pre-revolutionary France. In the reigning ideology of absolutism, the King was the only "public person," but over the second half of the $18^{\text {th }}$ century a new source of authority crept into politics. Across the political spectrum, the French elite hated the seemingly chaotic, fear-based, irrational party politics of England after 1688. And yet, they also articulated a desire for "liberty" of some sort, in opposition to the perceived despotism of the King's ministers, who spent the second half of the 18th century attempting to beat the regional parlements into submission. Simultaneously, another transfer occurred: from the early 18th century discourse concerning how an international "public" of Europe would judge the maneuvers of various absolutist regimes, to the possibility that a domestic "public" could discuss, and perhaps even judge, its own monarch — and especially the actions of his ministers. The result of this interwoven process was a thorough transformation of "public opinion," which came to stand for both "the people" and for rationality and enlightenment. ${ }^{17}$

\footnotetext{
${ }^{17}$ In 1750, Rousseau had used public opinion to refer to collective values and customs and the source of the social standing of individuals. In contrast, "From
} 
Baker's work was an iconic moment in the cultural turn in the historiography of the French Revolution, but recent work has extended his analysis to the material substrate of this new political object_-in particular the explosion of mass printing techniques, which combined with these relational meanings to make the new object possible. As Colin Jones (1996) argues, the Affiches (newspapers primarily driven by advertisements but prone to some news reporting as well, especially in the 1780s) "could claim to embody as well as represent that 'public opinion' whose importance recent historians have not been slow to emphasize" (Jones 1996: 39). Public opinion-an essential part of the cultural context of the 1780 s - thus came into being haltingly and through a highly varied assemblage of different sorts of stuff - signs, circulars, spoken gossip and debate among nobles, etc.

The invention of public opinion is a formation story, but it does not fit easily into the types we have outlined, and that we understand as well-established by sociological research. Later, public opinion would become, in part, a sociotechnical object. ${ }^{18}$ But in this case, the invention and manifestation of public

$1770 \ldots$ the term [began] also to take on connotations of the Enlightenment and to acquire a more explicitly political resonance" (Baker 1990: 187).

18 This is clear in studies of $20^{\text {th }}$ century politics (Igo 2007, Osborne and Rose 1999, Perrin and McFarland 2011); Igo (2011) outlines the early development of survey research in a way that would clearly be considered a formation story, suggesting strongly that "public opinion" was reshaped into something that could be studied via certain instruments, and raising the issue of the relationship of such objects of modern social science techniques, a key point of the larger volume 
opinion was a driver of a "major shift in French culture" and becomes part of the "cultural origins of the French Revolution."

Note, however, that the importance of change in broad cultural formations is not usually understood in this way. Often, their explication is thought of as nonexplanatory, an interpretation of "epistemes" (Foucault 1971) or of symbolic landscapes in a way that is detached from causal social science (Geertz 2000). Alternately, via concepts designed to capture the direct effects of culture on action, such as values, culture can be thought of in forcing cause terms, though the scope and utility of such a formulation is hotly debated. If, as Orlando Patterson (2014: 2) has recently noted, cultural sociology suffers from, at one extreme, "the dogmatic rejection of causal explanations, and, at the other, explanatory evasiveness more generally," then perhaps this is because explanations that evoke cultural representations are especially likely to exceed the existing concepts of causality present in sociology. Without proposing a single resolution to debates about culture and causality here, we submit that some research questions that invoke the question of culture might be well addressed by looking closely at

(Camic, Gross, and Lamont 2011). However, in Baker's study, the formation of public opinion is not really a study of technique so much as it is a way to understand the fundamental cultural shift of the late 18th century in terms that can be traced empirically. 
formation as we have outlined it. ${ }^{19}$ Certainly this is what the historiography of the French Revolution would suggest.

\section{5: Discussion}

If we see formation stories as causal, we can then start to understand them as a specific genre of sociological argument, replete with an accepted set of questions, attendant methodological debates, exemplar cases, and an evolving theoretical toolkit. This conceptualization leads to insights about what debates about formation stories might look like, if they were recognized as such.

We can begin by noting how the different formation stories presented above share a common insight, namely, that the "object," "actor" or "organization" that sociologists have worked to understand as a part of forcing cause relations has particular qualities and properties due to the way in which it was formed. Note here that, in addition to being causal claims in their own right, formation stories establish empirically the boundaries of forcing cause claims. In essence, they offer an empirical, historically traceable reason why the scope conditions of a given analysis are what they are. So, one implication of our account is that sociology should be much more concerned with the history of its own objects of study, accounting for them historically rather than defining them according to its own conventions.

\footnotetext{
${ }^{19}$ For a similar argument, framed in terms of "landscapes of meaning", see Reed 2011: 161-162.
} 
Thus, formation stories provide a useful complement to the idea of "pathdependence," now widely understood as part of historical sociology. In path dependence, a contingent choice of institutional formation, object, or type of relationship A from the set $\{A, B, C \ldots\}$ at time $t_{1}$ causes $A$ to lock in for time $t_{2}$, $t_{3}, t_{4}, \ldots$, thus generating a sequence that can be analyzed in terms of the mechanisms of reproduction that create the "inertia" that we recognize as pathdependence (Mahoney 2000). Formation stories, on the other hand, help explain how $\mathrm{A}, \mathrm{B}$, and $\mathrm{C}$ came into existence in the first place, and tell us about the properties that those objects have as a result of the historical process of assemblage.

If formation stories are to serve as another kind of causal historical account complementary to path-dependence, much more debate is needed about what makes a particular formation story persuasive. In particular, we need methodological discussion of what constitutes a compelling counterfactual claim in formation stories. For a formation story to be taken as a causal story, the author of the claim must show plausible other ways the formation could have gone, and then account well for the difference between the plausible and the actual within the case under study. ${ }^{20}$ This difference is the primary difference that needs to be

\footnotetext{
${ }^{20}$ It is worth noting that, despite the great deal of rhetoric surrounding actornetwork theory that valorizes description and attacks standard forms of sociological and "social" explanation, Latour himself affirms these sorts of contrasts as essential to the operation whereby causes are assessed. In his
} 
explained in a formation story-qua-sociological claim. Note, however, that to even establish such a difference, the investigator must use evidence from the case under study to distinguish the merely possible from the plausible (on the idea of plausible alternatives, see Lewis 1973, Hawthorn 1993). This process, and debate about it, is quite familiar to historians, but it is not as commonly foregrounded in in-depth case studies in sociology. Explicit attention to counterfactuals would be an important step forward because as of now - perhaps because they are not usually taken as causal claims at all - formation stories tend not to make their counterfactuals available.

These consequences of understanding formation stories as causal are epistemological. There is also an ontological implication to adding forming stories to our causal repertoire: formation stories show that the causal powers of the social world are never "fully and finally formed." The objects, kinds, and forces of the social world are not raised in a historical garden of Eden, and then set free into the world of causality once they have matured, to forever participate

provocative discussion on construction, he notes that 'the 'making of' any enterprise-films, skyscrapers, facts political meetings, initiation rituals, haute couture, cooking... provides a rare glimpse of what it is for a thing to emerge out of inexistence by adding to any existing entity its time dimension. Even more important, when you are guided to any construction site you are experiencing the troubling and exhilarating feeling that things could be different, or at least that they could still fail." (Latour 2005: 89, emphasis in original) This is a somewhat melodramatic version of the argument presented here - that to get formations stories as causal stories, one must distinguish the plausible from the possible, and then explain the difference between the actual and the plausible by effectively tracing the history of the formation of an object. 
in the regularized causality of a well-ordered universe. Rather they are constantly available for, and sometimes subject to, intense revision, reassociation, and so on. Having been formed, they can be transformed. As such, their "objectness" is really a relative stability of associations, not a timeless property (Latour 1999). ${ }^{21}$

Although we agree with actor-network theory on many counts, we part ways on an important point of emphasis. Latour (2005:107) hightlights the distinction between translation and transportation, using explicitly causal language: "all the actors we are going to deploy might be associated in such a way that they make others do things. This is done not by transporting a force that would remain the same throughout as some sort of faithful intermediary, but by generating transformations manifested by the many unexpected events triggered in the other mediators that follow them along the line." In our terms, Latour is expressing a rather strong preference for those causes in the world that display a certain amount of eventfulness, and downplaying the role of forcing causes. We see no reason to automatically assume "mediation" predominates over "intermediation" in any given context. Indeed the preference for one over the other, if enacted a priori, limits empirical research. We would instead urge sociologists to explore the ways in which formation stories bound forcing causes, without committing to one being "more important" than another. The importance

${ }^{21}$ This point about the potential reformation of objects is also expressed in actornetwork Theory via a preference for "performative" rather than "ostensive" definitions of objects (Latour 2005: 34). 
of different genres of causal explanation should depend pragmatically on the research question at hand, and empirically, on the extent to which actors, objects, fields, and cultural atmospheres are stable enough to be usefully modeled with forcing cause approaches.

Together, these epistemological and ontological implications of understanding formation stories as part of the causal repertoire of sociological research imply that generalizing from formation stories will proceed differently from forcing cause accounts. Specifically, we suggest that analogy (see Vaughan 2004, 2014) is a particularly appropriate mode of generalization for formation stories; rather than the "modularity" that is expected of treatment-style causal reasoning and some mechanistic approaches (Woodward 2003: 336-339), in which the very possibility of making a causal claim is premised on being able to add, remove or tweak a discrete element of a causal system while leaving the rest of the system intact. Instead, formation stories are claims about a messy assemblage of links, entities, etc. An assemblage that produces an object or kind cannot be parsed into an aggregation of "lower level" mechanisms, which then can be abstracted and transposed directly to other places and times. However, a well-established formation story can be carried beyond the context of its initial utility by analogical thinking as a form of generalization.

For example, the "performativity of economics" (Callon 1998, MacKenzie and Millo 2003) and Hacking's (1995) "looping effects" offer two examples of 
generalizable formation stories. While the details of how economic theories intersect with political and technological developments to shape markets will vary dramatically from case to case, the observation that particular modes of thought are especially generative focuses our attention on that potential cause and at least suggests its potential causal weight in new contexts. Similarly, having shown in a handful of contexts the dynamic processes by which new diagnoses of mental illness shape the behaviors of those diagnosed, and their social networks and political communities, which in turn reshape the meaning of the diagnosis, we come to expect that similar processes will occur in other similar cases, even as we recognize the particularity of the conjunctions of ideas, people, organizations, and tools that constitute specific assemblages like "autism" or "multiple personality disorder" (Hacking 2007, Eyal 2010).

\section{Conclusion}

The history of sociological theory is full of arguments for and against "reducing" sociological explanation to various core entities or actors and thus also with attempts to transcend the sacred oppositions of individual and social structure, consciousness and culture, and so on. Contemporary versions of this argument have focused carefully on the particulars of how causal images of the world can be mobilized in research. This renewed interest in getting causality right has led to important links between the philosophy of the social sciences, sociological theory, and sociological research, including extensive investigation 
into the foundations of three well-known approaches to causality based on the rigorous conceptualization of variables, treatments, and mechanisms.

One might hope, for parsimony's sake, that these versions of "forcing causality" could eventually be extended to cover formations stories - that, in other words, formation stories are just accounts of important strands of causal history that have not been adequately parsed into forcing causes yet, either from lack of knowledge, lack of trying, or lack of adequate theory. A similar, though less broad objection was recently registered in the pages of Sociological Theory, when Kuorikoski and Pöyhönen (2012) argued that Hacking's looping kinds are in fact reducible to a mechanistic framework. This argument was then used, somewhat predictably, to hammer "social constructionism," a position framed in opposition to one that studies "an empirical causal phenomenon within society" (Kuorikoski and Pöyhönen 2012:195)

We contend that formation stories are real, consequential, and empirically traceable, but not comprehensible in the terms of mechanistic, treatment, or variable approaches to causality. Thus this sort of opposition-between constructionism and empirical causal phenomenon — does not make sense. Instead, we should develop better, more elaborated, and more varied approaches to causality for sociology. One way to do this is to pursue a deeper understanding of formation stories. By accepting that arguments that trace the messy history of formation are, in fact, causal arguments, sociologists would be positioned to 
develop methods for telling better formation stories, and better, more general, theories for understanding how formation happens. We see such an expansion as preferable to two alternatives: first, that sociologists continue to try to pack formation stories into one of the three well-established versions of forcing causality, or, second, that sociologists simply treat accounts of formation as "interpretive," "descriptive" or "merely historical," in other words, as non-causal.

The essence of our argument can perhaps be captured via an oversimplified metaphor. Piecing together social causality out of variables, treatments, and mechanisms has been, to this point, something like understanding the causal interactions within a massively difficult and highly complex Rube Goldberg machine. But how did the pieces of that machine - the marbles, ramps, springs, and so on - come to be what they are? Some versions of the philosophy of social science argue that the intellectual operation by which one "reduces" the properties of a marble to its molecular components can be imitated in sociology. In contrast, our argument is that in sociology, the equivalent to "the marbles" come to have their existence, shape, and properties not through the aggregation of well-defined and fixed lower-level entities, but rather through eventful histories, assemblages of heterogeneous stuff, and meaningful representations. How did there come to be capitalists? How did options become so important in modern financial markets? Why did something called public opinion, emerge, take shape, and become essential for certain forms of legitimate domination? By 
conceptualizing these stories as causal processes of formation, we can come closer to explaining why social reality is the way it is. 


\section{References}

Abbott, Andrew. 1988. "Transcending General Linear Reality." Sociological Theory 6(2):169-86.

Abbott, Andrew. 1995. "Things of boundaries." Social Research 62(4):857.

Abbott, Andrew. 2001. Time Matters: On Theory and Method. University Of Chicago Press.

Abbott, Andrew. 2007. "Against Narrative: A Preface to Lyrical Sociology*." Sociological Theory 25(1):67-99.

Alexander, Jeffrey. 1981. Theoretical Logic in Sociology, Volume 1. Berkeley, CA: University of California.

Alexander, Jeffrey and Steven Seidman. 2008. "Introduction" in The New Social Theory Reader. New York: Routledge.

Archer, Margaret S., Roy Bhaskar, Andrew Collier, Tony Lawson, and Alan Norrie, editors. 1998. Critical Realism: Essential Readings. Routledge.

Baker, K. 1990. Inventing the French Revolution. Cambridge University Press.

Barman, Emily. 2013. "Classificatory Struggles in the Nonprofit Sector: The Formation of the National Taxonomy of Exempt Entities, 1969-1987." Social Science History 37(1):103-41.

Benford, Robert D., and David A. Snow. 2000. "Framing processes and social movements: An overview and assessment." Annual review of sociology 611-39.

Bhaskar, Roy. 2008. A Realist Theory of Science. Taylor \& Francis.

Bijker, Wiebe E. 1997. Of Bicycles, Bakelites, and Bulbs: Toward a Theory of Sociotechnical Change. Cambridge, Mass.: The MIT Press.

Blau, Peter M., and Otis Dudley Duncan. 1967. The American Occupational Structure. New York: Wiley \& Sons.

Bourdieu, Pierre. 1984. Distinction: A Social Critique of the Judgement of Taste. Harvard University Press.

Bourdieu, Pierre. 1990. The Logic of Practice. Stanford University Press.

Bourdieu, Pierre, Jean-Claude Chamboredon, Jean-Claude Passeron, and Beate Krais. 1991. The craft of sociology. Walter de Gruyter.

Calhoun, Craig. 1998. "Explanation in Historical Sociology: Narrative, General Theory, and Historically Specific Theory." The American Journal of Sociology 104 (3): 846-871.

Callon, Michel, and Bruno Latour. 1981. "Unscrewing the big Leviathan." Advances in social theory and methodology: 277-303.

Callon, Michel. 1998. "Introduction: the embeddedness of economic markets in economics." Pp. 1-57 in The Laws of the Markets. Oxford: WileyBlackwell. 
Camic, Charles, Neil Gross and Michele Lamont, editors. 2011. Social Knowledge in the Making. Chicago: University of Chicago Press.

Clemens, Elisabeth S. 2007. "Toward a Historicized Sociology: Theorizing Events, Processes, and Emergence.” Annual Review of Sociology 33: 527549.

Coleman, James Samuel. 1994. Foundations of Social Theory. Harvard University Press.

Darnton, R. 1996. The Forbidden Best-Sellers of Pre-Revolutionary France. New York: W.W. Norton \& Co.

Daston, Lorraine. 2000. Biographies of Scientific Objects. University Of Chicago Press.

Dimaggio, Paul, and Walter W. Powell. 1983. “The Iron Cage Revisited: Institutional Isomorphism and Collective Rationality in Organizational Fields." American Sociological Review 48:147-60.

Eyal, Gil. 2010. The Autism Matrix. Polity.

Eyal, Gil. 2013. "For a Sociology of Expertise: The Social Origins of the Autism Epidemic.” American Journal of Sociology 118(4):863-907.

Eyal, Gil, and Larissa Buchholz. 2010. "From the Sociology of Intellectuals to the Sociology of Interventions.” Annual Review of Sociology 36(1):117-37.

Fligstein, Neil, and Doug McAdam. 2011. "Toward a General Theory of Strategic Action Fields." Sociological Theory 29(1):1-26.

Foucault, Michel. 1971. The Order of Things: An Archaeology of the Human Sciences. Pantheon.

Foucault, Michel. 1972. The Archaeology of Knowledge. Pantheon.

Foucault, Michel. 1977. Discipline \& Punish: The Birth of the Prison. Pantheon.

Fourcade, Marion, and Kieran Healy. 2013. "Classification situations: Lifechances in the neoliberal era." Accounting, Organizations and Society 38(8):559-72.

Geertz, Clifford. 2000. The Interpretation of Cultures. Basic Books.

Giddens, Anthony. 1979. Central Problems in Social Theory: Action, Structure, and Contradiction in Social Analysis. Berkeley, CA: University of California Press.

Goertz, Gary. 2006. Social science concepts: A user's guide. Princeton University Press.

Goldthorpe, John H. 2001. "Causation, Statistics, and Sociology.” European Sociological Review 17(1):1-20. 
Gorski, Philip. 2009. “Social 'mechanisms' and Comparative-historical Sociology: A Critical Realist Proposal.” In Peter Hedstrom and Bjorn Wittrock, editors, The Frontiers of Sociology. Brill.

Groff, Ruth and John Greco, eds. 2013. Powers and Capacities in Philosophy: the New Aristotelianism. New York: Routledge.

Gross, Neil. 2006. "Comment on Searle." Anthropological Theory. 6 (1): 45-56. Hacking, Ian. 1995. "The looping effects of human kinds." Causal cognition: A multidisciplinary approach 351-83.

Hacking, Ian. 2000. The Social Construction of What? Harvard University Press. Hacking, Ian. 2002. Historical Ontology. Harvard University Press.

Hacking, Ian. 2007. "Kinds of people: moving targets." P. 285 in Proceedings of the British Academy, vol. 151.

Hawthorn, Geoffrey. 1993. Plausible Worlds: Possibility and Understanding in History and the Social Sciences. New York: Cambridge University Press.

Heckman, James J. 2005. "The scientific model of causality." Sociological methodology 35(1):1-97.

Hedstrom, Peter and Peter Bearman. 2009 "What is Analytical Sociology All About? An Introductory Essay," in The Oxford Handbook of Analytical Sociology. New York: Oxford.

Hedstrom, Peter, and Petri Ylikoski. 2010. "Causal Mechanisms in the Social Sciences.” Annual Review of Sociology 36(1):49-67.

Hedstrom, Peter. 2005. Dissecting the Social: On the Principles of Analytical Sociology. Cambridge University Press.

Hempel, Carl. 1965. Aspects of Scientific Explanation and Other Essays in the Philosophy of Science. New York: Free Press.

Holland, Paul W. 1986. "Statistics and Causal Inference." Journal of the American Statistical Association 81(396):945-60.

Igo, Sarah E. 2007. The Averaged American: Surveys, Citizens, and the Making of a Mass Public. Harvard University Press.

Igo, Sarah E. 2011. "Subjects of Persuasion: Survey Research as a Solicitous Science; or The Public Relations of the Polls.” Pp. 285-306 in Charles Camic, Neil Gross, and Michele Lamont, editors. Social Knowledge in the Making. Chicago: University of Chicago Press.

Jones, Colin. 1996. "The Great Chain of Buying: Medical Advertisement, the Bourgeois Public Sphere, and the Origins of the French Revolution." The American Historical Review 101 (1): 13-40.

Kirkland, Anna. 2012. "Credibility battles in the autism litigation." Social Studies of Science 42(2):237-61.

Kiser, Edgar, and Michael Hechter. 1991. "The Role of General Theory in Comparative-Historical Sociology.” American Journal of Sociology 97 
(1): $1-30$.

Knight, Carly R., and Christopher Winship. 2013. "The causal implications of mechanistic thinking: Identification using directed acyclic graphs (DAGs)." Pp. 275-99 in Handbook of Causal Analysis for Social Research. Springer.

Kuorikoski, Jaakko, and Samuli Pöyhönen. 2012. "Looping Kinds and Social Mechanisms." Sociological Theory 30(3):187-205.

Lakoff, George, and Mark Johnson. 1999. Philosophy in the Flesh: The Embodied Mind and Its Challenge to Western Thought. Basic Books.

Latour, Bruno. 1999. Pandora's Hope: Essays on the Reality of Science Studies. Harvard University Press.

Latour, Bruno. 2007. Reassembling the Social: An Introduction to Actor-NetworkTheory. Oxford University Press, USA.

Lewis, David. 1973. "Causation.” Journal of Philosophy 70(17): 556-567.

Liu, Ka-Yuet, Marissa King, and Peter Bearman. 2010. "Social Influence and the Autism Epidemic.” American Journal of Sociology 115(5):1387-1434.

Luker, Kristin. 2008. Salsa Dancing into the Social Sciences: Research in an Age of Info-glut. Harvard University Press.

MacKenzie, Donald, and Yuval Millo. 2003. "Constructing a Market, Performing Theory: The Historical Sociology of a Financial Derivatives Exchange." American Journal of Sociology 109(1):107-45.

MacKenzie, Donald, Daniel Beunza, Yuval Millo, and Juan Pablo Pardo-Guerra. 2012. "Drilling through the Allegheny Mountains: liquidity, materiality and high-frequency trading." Journal of cultural economy 5(3):279-96.

Mahoney, James. 2000. "Path Dependence in Historical Sociology." Theory and Society 29(4):507-48.

Mahoney, James. 2001. "Beyond Correlational Analysis." Sociological Forum. 16(3): 575-593.

Mahoney, James. 2012. "The Logic of Process Tracing Tests in the Social Sciences." Sociological Methods \& Research 41(4):570-97.

Mahoney, James and Dietrich Rueschemeyer. 2003. "Comparative Historical Analysis: Achievements and Agendas." In Comparative Historical Analysis in the Social Sciences. Edited by James Mahoney and Dietrich Rueschemeyer. New York: Cambridge University Press.

Medvetz, Thomas. 2012. Think Tanks in America. University Of Chicago Press. Meyer, John W., and Ronald L. Jepperson. 2000. "The 'Actors' of Modern Society: The Cultural Construction of Social Agency.” Sociological Theory 18(1):100-120. 
Mirowski, P., and E. Nik-Khah. 2007. "Markets made flesh: performativity, and a problem in science studies, augmented with consideration of the FCC auctions." Do Economists Make Markets 190-224.

Mitchell, T. 1998. "Fixing the Economy." Cultural Studies 12(1):82-101.

Morgan, Stephen L., and Christopher Winship. 2007. Counterfactuals and Causal Inference: Methods and Principles for Social Research. 1st edition. Cambridge University Press.

Morgan, Stephen L., and Christopher Winship. 2014. Counterfactuals and Causal Inference: Methods and Principles for Social Research. 2nd edition. Cambridge University Press.

Oakes, Guy. 1990. Weber and Rickert: Concept Formation in the Cultural Sciences. Mit Press.

Omi, Michael, and Howard Winant. 1994. Racial Formation in the United States: From the 1960s to the 1990s. Psychology Press.

Osborne, Thomas, and Nikolas Rose. 1999. "Do the social sciences create phenomena?: the example of public opinion research." The British Journal of Sociology 50(3):367-96.

Outhwaite, William. 2010. Concept Formation in Social Science. Routledge.

Padgett, John F., and Walter W. Powell. 2012. The Emergence of Organizations and Markets. Princeton University Press.

Patterson, Orlando. 2014. "Making Sense of Culture." Annual Review of Sociology 40(1):1-30.

Perrin, Andrew J., and Katherine McFarland. 2011. "Social Theory and Public Opinion." Annual Review of Sociology 37(1):87-107.

Polletta, Francesca, and James M. Jasper. 2001. "Collective identity and social movements." Annual review of Sociology 283-305.

Quadagno, Jill, and Stan Knapp. 1992. "Have Historical Sociologists Forsaken Theory? Thoughts on the History/Theory Relationship." Sociological Methods and Research 20 (4): 481-507.

Ragin, Charles C. 1989. The Comparative Method: Moving Beyond Qualitative and Quantitative Strategies. Univ of California Press.

Reed, Isaac. 2011. Interpretation and Social Knowledge. University of Chicago Press.

Ringer, Fritz K. 1997. Max Weber's Methodology. Harvard University Press.

Sartori, Giovanni. 1970. "Concept misformation in comparative politics." The American Political Science Review 1033-53.

Savage, Mike. 2009. "Contemporary Sociology and the Challenge of Descriptive Assemblage.” European Journal of Social Theory 12 (1): 155-174.

Sayes, Edwin. 2014. "Actor-Network Theory and methodology: Just what does it mean to say that nonhumans have agency?" Social Studies of Science 44(1):134-49. 
Searle, John. 1995. The Construction of Social Reality. New York: Simon and Schuster.

Sen, Maya and Omar Wasow. 2014. "Reconciling Race and Causation: Designs to Estimate Effects of Seemingly Immutable Characteristics.” Working paper.

Sewell, William H., Jr. 2005. "Three Temporalities: Toward an Eventful Sociology.” Pp. 81-123 in Logics of History: Social Theory and Social Transformation. University of Chicago Press.

Somers, Margaret R. 1995. "What's Political or Cultural about Political Culture and the Public Sphere? Toward an Historical Sociology of Concept Formation." Sociological Theory 13(2):113-44.

Star, Susan Leigh. 2010. "This is Not a Boundary Object: Reflections on the Origin of a Concept." Science, Technology \& Human Values 35(5):60117.

Steinmetz, George. 1998. "Critical Realism and Historical Sociology. A Review Article." Comparative Studies in Society and History 40 (01): 170-186.

Steinmetz, George. 2004. "Odious comparisons: Incommensurability, the case study, and 'small N's' in sociology." Sociological Theory 22(3):371-400.

Steinmetz, George. 2007a. The Devil's Handwriting: Precoloniality and the German Colonial State in Qingdao, Samoa, and Southwest Africa. University of Chicago Press.

Steinmetz, George. 2007b. "Fordism and the Positivist Revenant: Response to Burris, Riley, and Fourcade." Social Science History 31(1):127-52.

Stinchcombe, Arthur L. 1991. "The Conditions of Fruitfulness of Theorizing About Mechanisms in Social Science." Philosophy of the Social Sciences 21(3):367-88.

Tilly, Charles. 1995a. "To Explain Political Processes.” American Journal of Sociology: 1594-1610.

Tilly, Charles. 1995b. European Revolutions, 1492-1992. Cambridge, MA: Blackwell.

Tilly, Charles. 2004. "Social Boundary Mechanisms." Philosophy of the Social Sciences 34(2):211-36.

Vaughan, Diane. 2004. "Theorizing Disaster Analogy, Historical Ethnography, and the Challenger Accident." Ethnography 5 (3) (September 1): 315-347.

Vaughan, Diane. 2014. "Analogy, Cases, and Comparative Social Organization." In Theorizing in Social Science. Edited by Richard Swedberg. Stanford University Press.

Weber, Max. 1949. "Objectivity in Social Science and Social Policy." In The Methodology of the Social Sciences. Edited by E.A. Shils and H.A. Finch. The Free Press. 
Weber, Max. 1958. From Max Weber: Essays in Sociology. Oxford University Press.

White, Harrison C. 2008. Identity and control: How social formations emerge. Princeton University Press.

Woodward, James. 2003. Making Things Happen: A Theory of Causal Explanation. New York: Oxford University Press. 